\title{
Automatic Categorization of Human-Coded and Evolved CoreWar Warriors
}

\author{
Nenad Tomašev, Doni Pracner, Miloš Radovanović, and Mirjana Ivanović \\ University of Novi Sad \\ Faculty of Science, Department of Mathematics and Informatics \\ Trg D. Obradovića 4, 21000 Novi Sad, Serbia \\ tomasev@nspoint.net, doni@neobee.net, \{radacha,mira\}@im.ns.ac.yu
}

\begin{abstract}
CoreWar is a computer simulation devised in the 1980s where programs loaded into a virtual memory array compete for control over the virtual machine. These programs are written in a special-purpose assembly language called Redcode and referred to as warriors. A great variety of environments and battle strategies have emerged over the years, leading to formation of different warrior types. This paper deals with the problem of automatic warrior categorization, presenting results of classification based on several approaches to warrior representation, and offering insight into ambiguities concerning the identification of strategic classes. Over 600 human-coded warriors were annotated, forming a training set for classification. Several major classifiers were used, SVMs proving to be the most reliable, reaching accuracy of $84 \%$. Classification of an evolved warrior set using the trained classifiers was also conducted. The obtained results proved helpful in outlining the issues with both automatic and manual Redcode program categorization.
\end{abstract}

\section{Introduction}

CoreWar was introduced by A. K. Dewdney in 1984 in an article published in Scientific American [1]. It was based on a game called Darwin developed in Bell Labs in 1960, devised by Victor Vyssotsky, Robert Morris Sr. and Dennis Richie. In CoreWar, several programs, referred to as warriors, attempt to survive in a looping memory array, avoiding attacks and at the same time trying to eliminate the opposition. The warrior that takes complete control of the process queue wins the battle. A match between two warriors consists of a number of such battles, each time varying the initial positioning in the memory array which is referred to as the core.

Several automatic warrior generators have been created so far, utilizing evolutionary algorithms to create functioning warriors out of sets of randomly generated code sequences. Performance evaluation in warrior generation is usually done via testing against some predetermined benchmark warrior set of considerable size. In evolvers, when a lot of new warriors are being constructed in each generation, determining fitness becomes a very time demanding process.

Automatic warrior categorization could be very useful in CoreWar evolutionary software in order to design control mechanisms for mutation rate adjustment. High mutation rates allow the creation of a greater variety of forms, while the low mutation 
rates instigate convergence of the generation pools to the fittest among the generated types. However, the current lack of automatic categorization renders diversity supervision practically impossible. Some warriors may be easy to categorize manually by the combination of strategic components in their code, but finding clear distinctions between warrior types is generally not an easy task.

The goal of the research presented in this paper is to explore the possibilities for automatic warrior categorization using representations based on syntax analysis and benchmark scores. The former may quickly and easily be calculated, while the latter are an essential part of fitness evaluation in CoreWar evolvers. The addressed issues include choosing warrior types from a plethora of possible distinctions depending on the desired level of abstraction, specifying the representations, manually categorizing a warrior set, conducting automatic classification, and testing it on both human-coded and evolved warrior sets. Our main intention was to spot the obstacles in the categorization process, so that further improvements to the representations could be made and, more importantly, to assess the feasibility of automatic warrior categorization. This project is the first attempt to achieve the above mentioned goal using supervised machine learning methods, and also the first to introduce a fully labeled warrior dataset.

The rest of the paper is organized as follows. Section 2 explains the essentials of CoreWar and the Redcode language, while Section 3 discusses possibilities for representing warriors in a form suitable for analysis. Section 4 deals with the issues related to both human-coded and evolved warrior datasets used in this research. The analysis of categorization itself is given in Section 5 The last section provides a summary of the conclusions together with plans for future work.

\section{CoreWar}

CoreWar is a computer simulation where programs compete in a virtual cyclic memory array. These programs, referred to as warriors, are written in an assembly language called Redcode. Warrior confrontation takes place in a virtual memory array called the core which is wrapped around so that the successor of the last address in the core is the first one. Execution of instructions and management of threads is performed by the memory array redcode simulator (MARS).

A warrior's goal in most competitions is to take complete control of the core by forcing all its opponents to eliminate their threads of execution from the process queue. Warriors can read from the core, write to the core, perform basic arithmetic instructions, create new threads, mutate, go through numerous stages in their ontogeny, copy themselves, actively search for their opponents, etc.

Competitions are held regularly on several Internet servers. Corewar leagues are commonly referred to as hills. There are several important parameters defining these standard competitions, namely: core size, maximal battle duration, number of threads allowed per warrior, warrior size restrictions, etc. The most popular hill is certainly the 94nop hill, which is the presumed setting for warriors considered in this paper.

The Redcode language. Redcode is currently the default language for writing CoreWar warriors. It contains 19 instructions, 7 instruction modifiers and 8 addressing modes. Each command consists of an instruction name, followed by the instruction modifier, 
A-field addressing mode, A-field value, B-field addressing mode and B-field value. The more important instructions include DAT, which is used both to store data and remove the thread executing it from the process queue; the copying instruction MOV; arithmetic instructions ADD, SUB, MUL, DIV and MOD; unconditional jump instruction JMP and conditional jumps JMZ, JMN and DJN; and the thread-creating instruction SPL. There are many combinations of the mentioned elements, more precisely 8512 . CORESIZE ${ }^{2}$. The set of all possible Redcode programs in the standard setting is of cardinality $100 \cdot\left(544768000000^{100}\right)$. A description of Redcode is available in [2].

Warrior types. Contemporary warriors are highly sophisticated, a result of over two decades of continuous improvements over the basic ideas, and occasional ascension of new concepts. Most of them represent combinations of several strategic elements. Some common strategic concepts are summarized below.

Imps are among the simplest of components, yet quite often used due to the fact that disposing of them is costly in terms of time and space. Imps consist only of MOV instructions, copying themselves through the core, commonly forming structures known as rings and spirals. Bombing is a process of copying some predetermined instructions throughout the core with the intention of overwriting a part of the opponent's code. Replication is a process performed by warrior components constantly copying themselves and creating new threads to run the copies. Core clearing is a process of sequential overwriting of the core with some predetermined instruction. Scanning denotes heuristically searching for opponent's code by comparing pairs of instructions or instruction fields. Bootstrapping is a process of quickly copying essential components away from the original code to avoid detection. Quickscan is a component performing exceptionally fast scanning at the start of the simulation, trying to locate enemy code in an early stage and disable it before it bootstraps and activates its components.

For the purposes of categorization in this paper, a relatively modest number of 13 warrior types has been selected to represent the strategic abundance of CoreWar. The considered warrior types are given in Table 1

\section{Warrior Representation}

One of the main issues in automatic CoreWar warrior categorization is certainly representing warriors in a form suitable for analysis. The code itself can be viewed as a genotype, while the associated behavior in a certain core corresponds to the phenotype of a warrior. Same warrior code can display different properties in different environments and even belong to different warrior types in the respective core settings! Therefore, it is the emergent behavior that outlines the category generalizing the strategic concepts of a warrior in a certain environment. In the rest of this paper the concept of warrior types will be regarded in this manner, only relative to the 94nop setting.

The question arises whether it is possible to draw conclusions about warrior phenotype given the parameters of the considered environment, based on observations of code alone. If it were possible to classify a warrior based on a representation derived from syntax analysis, such a process would be favorable in terms of execution time, and therefore preferable for use in systems performing a lot of calculations, e.g. evolvers. 
Table 1. Warrior categories

\begin{tabular}{ll}
\hline Type & \\
\hline cds & Clear-directing scanners \\
clr & Warriors basing their activity on clearing the core \\
clrwi & Core-clearing warriors using imp components \\
evo & Evolved warriors, a category denoting all automatically created warriors that do not \\
& resemble human-coded warriors enough to be considered one of the other types \\
onesh & Oneshots - a special class of scanners, focusing on the first potential threat \\
pap & Replicators, also called papers, according to the stone/paper/scissor analogy \\
pwi & Replicators that also use imps \\
pws & Replicators that also use stones \\
sabi & Stones accompanied by both A-field and B-field imps \\
sai & Stones accompanied by A-field imps \\
sbi & Stones accompanied by B-field imps \\
scn & Scanners other than clear-directing scanners and oneshots. All three classes to- \\
& gether are referred to as scissors \\
stn & Stones are warriors utilizing a bombing strategy. Their name is derived from the \\
& stone/paper/scissor analogy \\
\hline
\end{tabular}

In the first phase, a simple syntax-based representation was evaluated comprised of frequencies of appearance of instruction names in warrior code, and also of some characteristic instruction pairs, namely SPLMOV, MOVJMP, MOVDJN, MOVADD, MOVSUB, SEQSNE, SNEJMP, and SEQSLT. A boolean flag Impspec was added to carry information about the potential imp presence within a warrior. We shall denote this representation static. It total, it consists of 26 continuous, and one boolean attribute.

In the second phase, a representation formed by benchmark scores was used. These data are usually available in automatic warrior generators, due to necessity of performing fitness estimation. This representation consists of win and loss percentages of the tested warrior against each of the benchmark warriors, and will be referred to as the score representation. The benchmark was comprised of 30 carefully chosen warriors, accounting for 60 continuous attributes for this representation.

After both mentioned representations were tested, a hybrid representation combining the former was used (denoted combined). It was additionally attempted to extend the representations with another boolean feature named Qspec, indicating the presence of a quickscan component within a warrior. This attribute will be treated separately since we do not yet have a satisfactory means of automatically determining its value.

The static representation suffers heavily from its inability to distinguish code that will be executed during the simulation from decoys. There was an attempt in the past to overcome this problem by observing frequencies of command executions during simulations [3]. This may seem to be a good solution, but it has its own pitfalls. In particular, when a warrior is placed alone in the core there is a strong possibility that some parts of its code will never be executed because no enemy is ever detected. If, on the other hand, a warrior is set to confront some other warriors, it will also be executing commands that other warriors might copy over its code. Syntax analysis does not fall prey to the described problem. 
Table 2. Class distribution of the h1c dataset

\begin{tabular}{cccccccccccccc}
\hline cds & clr & clrwi & evo & onesh & pap & pwi & pws & sabi & sai & sbi & scn & stn & Total \\
\hline 47 & 20 & 12 & 66 & 73 & 100 & 40 & 40 & 6 & 33 & 39 & 73 & 117 & 666 \\
\hline
\end{tabular}

\section{The Datasets}

Two warrior sets have been used in this research. The first one, denoted h1c, represents a subset of 94nop Königstuhl set [4]. All warriors were manually categorized. The dataset consists of 666 warriors (no pun intended), which are unfortunately not evenly distributed among the considered categories (Table2). The average warrior length in the dataset disregarding data storing instructions was 35, far below the 100 instruction limit imposed by the 94nop setting. Massive use of quickscanners as an early stage strategic component led to the minimalistic approach in coding to avoid early detection.

The considered evolved warrior set is a subset of the output generated by the CCAI evolver [5], which was written by Barkley Vowk from the University of Alberta in summer 2003. We have already subjected the complete CCAI output to clustering using the static representation in [2]. The respective size of that dataset is 4389 warriors.

\section{Warrior Categorization}

Classification was performed using the WEKA machine learning workbench [6]. The following classifiers were included in the experiments: SMO - an implementation of the sequential minimal optimization algorithm for training support vector machines [7], performing multi-class classification using a binary classifier for each pair of classes; MultilayerPerceptron (MLP) - a neural network classifier trained using backpropagation; J48 - a decision tree learner based on revision 8 of the C4.5 algorithm; NaiveBayes; BayesNet - a Bayesian network with automatically determined structure as the maximum weight spanning tree [8]; and IBk - which implements the classical k-nearest neighbor algorithm. We report results for SMO with the linear kernel and $C=1$, MLP trained in 500 epochs with one hidden layer containing $1 / 2$ of the total number of input and output nodes, NaiveBayes with supervised discretization used for handling continuous attributes, and IBk with $k=5$ neighbors and reciprocal distance weighing.

Categorization of human-coded warriors. Figure 1 summarizes the performance of the considered classifiers in experiments involving 10 runs of 10-fold cross-validation on the h1c dataset. The highest accuracy was exhibited by SMO, 84.26\%, on the representation including both static and score components, including specification of quickscanner presence. Generally, the use of the Qspec attribute only slightly improved the performance of classification with all representations, and its contribution could not be statistically verified using the corrected resampled t-test $(p=0.05)$.

Performance of all classifiers except for MLP was statistically verified as worse when compared to SMO on the complete (combined+Qspec) representation. NaiveBayes proved to be the worst among the tested classifying methods, reaching accuracy of only $69.7 \%$. Apparently, it had problems coping with the dependencies between 


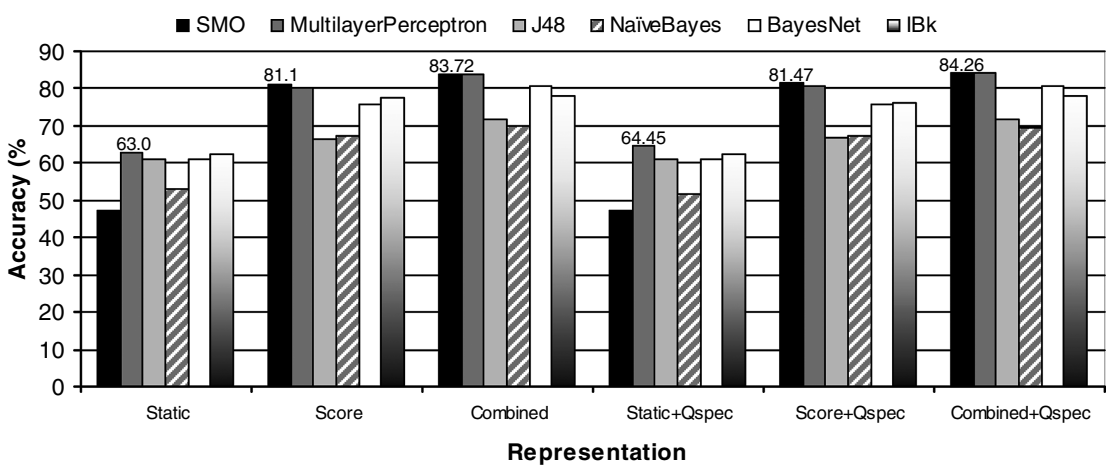

Fig. 1. Performance of various classifiers on the h1c warrior dataset, by representation

attributes, both static (concerning co-occurrences of instructions) and score-based (regarding the pairwise and other dependencies between win and loss percentages), which may be observed on the partial representations. This is corroborated by the bad performance of BayesNet when configured to work with the "naive" network structure (its accuracy is only slightly better than that of the the reported NaiveBayes).

The classification success rate varied respective to warrior categories in question. Replicators were easily recognized. Some scanners belonging to the scn class had been confused with stones. The scanners in question were mostly the less efficient ones, thus differing in crucial benchmark scores from the rest of their group, instead scoring similarly to a warrior subtype known as incendiary stones. The lowest accuracy was present in classification of warriors belonging to those categories represented by a small number of instances in the dataset, namely clr, clrwi, and sabi. It is also worth mentioning that most of the evolved warriors in h1c were correctly classified as evo.

Classifier accuracy on the complete representation was superior over isolated use of static features. Also, adding the static feature vector to the score representation improved classification significantly in cases of SMO, MLP, BayesNet and J48 classifiers.

Even though SMO performed best on score-based and combined representations, its accuracy of $47.15 \%$ on the static representation was significantly inferior to the performance of J48, BayesNet, IBk and MLP. This can be partially remedied by employing polynomial kernels of higher degree (4-6), with the performance being able to reach that of the best classifiers, but at the expense of worsening accuracy on score-based representations. The highest accuracy of classification on the static representation was achieved by MLP - 64.45\%.

Regardless of the apparent advantages of both score-based and combined representations over the static representation, the obtained results indicate that classification according to static features alone might be possible in the future if some modifications were made. First of all, according to the current static representation, it is absolutely impossible to distinguish A-field imps from B-field imps. That can easily be solved by adding new imp presence indicators. Also, it appears that the use of characteristic instruction pairs was insufficient for carrying information about the context in which instructions were used. Some solutions to that problem will be considered in Section 6 
Categorization of the evolved dataset. Generation 4 of the CCAI dataset was already clustered [2], so the categorization of that warrior set was meant both as a test of the reliability of trained classifiers and also to provide insight into the structure of the dataset and its clusters. Clustering had been done using the static representation, without Qspec. For purposes of classification in the current research, classifiers were trained on h1c with the score and combined representations, and tested on a random 400-warrior sample from the CCAI dataset which was manually labeled.

Evolved warriors usually differ from their human-coded adversaries. One of the main characteristics of evolved warriors is the presence junk code, i.e. instructions in the source code which actually never get executed. Evolved datasets consist mainly of mutation resistant forms - core-clearing warriors and replicators being the dominant types [2]. Also, such warriors rarely utilize advanced strategic tricks, which distinguishes them from analogous human-coded warrior types. However, the last generation of the CCAI set exhibits somewhat different properties, as the evolver was aimed at generating strong, competitive warrior forms. The final product was a famous warrior that defeated many human-coded opponents, appropriately named Machines Will Rule. Hence, warriors in the CCAI set tend to be stronger than typical evolved warriors and bear more resemblance to their human-coded counterparts.

Manual inspection detected only two classes in the 400-warrior sample of the CCAI set: pap and pwi. Papers were mostly using anti-imp core-clearing techniques, and achieved great scores against imp-type warriors in the benchmark. Imp-containing papers, however, were not nearly as well optimized and rarely benefited from the presence of defensive imp structures.

The following classifiers were tested: SMO, MLP, BayesNet, and IBk, with the results summarized in Table 3 It can be seen that the introduction of static features to the score-based representation does not yield consistent improvements as with the h1c dataset. On the contrary, it significantly degraded the performance of MLP and IBk classifiers. This is the result of noise introduced by junk code in the evolved warriors which contained mostly imp-specific and arithmetic instructions, helping MLP confuse many pap warriors with pws. The misclassification rate of most classifiers originated from pap being interpreted as sbi. Besides junk code, this can be attributed to the high resistance of the warriors in question to some common scanner attack techniques. On the other hand, static features continue to carry useful information, which is evident from the introduced improvement to the accuracy of SMO and BayesNet, and also from the increase in the ability of most classifiers to detect the minority pwi class.

All of the warriors from the sample misclassified by either of the classifiers as sbi or pwi using the combined representation were additionally examined. In $37 \%$ of instances an interesting structure was discovered, used by the replicators as a strong anti-imp feature, but also forming a sort of imp-like structure, thus enhancing defensive capabilities. Such pseudo-spirals were set up in a similar fashion to imp spirals, the difference being in the instruction used, namely MOV.I \#1169, \}2667. Apart from this interesting replicator subtype, core-clearing papers were quite frequent in the considered sample.

As for the syntactic clustering described in [2], we can now conclude that it was unable to detect the subtle differences between the two classes in the dataset, being misled by junk code within the warriors. However, this does not mean that the clustering 
Table 3. Categorization of a 400-warrior sample from generation 4 of the CCAI dataset, with class counts and accuracy for the score and combined representations

\begin{tabular}{|c|c|c|c|c|c|c|c|c|}
\hline Classifier & evo & pap & pwi & pws & sabi & sbi & stn & Accuracy (\%) \\
\hline & $\mathrm{scr} \mathrm{cmb}$ & $\mathrm{scr} \mathrm{cmb}$ & $\mathrm{scr} \mathrm{cmb}$ & $\mathrm{scr} \mathrm{cmb}$ & $\mathrm{scr} \mathrm{cmb}$ & $\mathrm{scr} \mathrm{cmb}$ & $\mathrm{scr} \mathrm{cmb}$ & $\mathrm{scr} \quad \mathrm{cmb}$ \\
\hline SMO & $\begin{array}{ll}18 \quad 0 \\
\end{array}$ & $345 \quad 359$ & $\begin{array}{ll}1 & 0\end{array}$ & $\begin{array}{ll}19 & 0\end{array}$ & \begin{tabular}{|ll}
0 & 0
\end{tabular} & $\begin{array}{ll}17 & 41\end{array}$ & $\begin{array}{ll}0 & 0\end{array}$ & 88.50 \\
\hline MLP & 11 & $322 \quad 217$ & 14 & 136 & 2 & 12 & 0 & 53.25 \\
\hline BayesNet & 0 & $391 \quad 392$ & 1 & 0 & 0 & 8 & 0 & 96.50 \\
\hline IBk & 0 & $386 \quad 359$ & 2 & 0 & 0 & 12 & 0 & 88.50 \\
\hline Manual & $\overline{0}$ & 392 & 8 & 0 & 0 & 0 & 0 & 100.00 \\
\hline
\end{tabular}

was not a good indicator of the diversity of warrior genotype, since junk code can also be combined in subsequent generations to produce working warriors.

\section{Conclusions and Future Work}

Recently, the attention of the CoreWar community shifted from devising new tricks in the existing strategies to exploring new settings, parameter optimization [9], and automatic warrior generation [5]. Quick and reliable warrior categorization would be of great importance in many such automated optimizing systems. The results obtained from this research indicate that automatic categorization is indeed possible to achieve, at least in the standard 94nop environment. However, further research is necessary in order to improve classification accuracy and possibly form a more universal categorization model, applicable to a wider range of environments. We believe that changing the static part of the representation alone may be enough to ensure the desired increase of accuracy. Adding new n-grams to the representation, as well as modifying and decomposing the Impspec feature would certainly improve static-based categorization, and probably the combined representation as well.

\section{References}

1. Dewdney, A.K.: Computer recreations: In the game called core war hostile programs engage in a battle of bits. Scientific American 250(5), 14-22 (1984)

2. Pracner, D., Tomašev, N., Radovanović, M., Ivanović, M.: Categorizing evolved CoreWar warriors using EM and attribute evaluation. In: Proc. MLDM'07, 5th Int. Conf. on Machine Learning and Data Mining in Patt. Recognition. LNAI, vol. 4571, Springer, Heidelberg (2007)

3. Varfar, W.: Wilfiz scores of warriors on the 94nop.

redcoder. sourceforge.net/?p=kepler-wilfiz

4. Birk, C.: CoreWar Koenigstuhl, Www. ociw. edu/ birk/COREWAR/koenigstuhl . html

5. Vowk, B.: CCAI.,www.math.ualberta.ca/ bvowk/corewar.html

6. Witten, I.H., Frank, E.: Data Mining: Practical Machine Learning Tools and Techniques, 2nd edn. Morgan Kaufmann Publishers, San Francisco (2005)

7. Platt, J.: Fast training of support vector machines using sequential minimal optimization. In: Advances in Kernel Methods - Support Vector Learning, MIT Press, Cambridge (1999)

8. Friedman, N., Geiger, D., Goldszmidt, M.: Bayesian network classifiers. Machine Learning 29(2-3), 131-163 (1997)

9. Zapf, S.: Optimax, www. corewar.info/optimax/ 